\title{
SHIFTS ACROSS TROPHIC LEVELS AS EARLY WARNING SIGNALS OF COPPER SULFATE IMPACTS IN PLANKTON COMMUNITIES
}

\author{
ANA I. DEL ARCO ${ }^{1}$ - FRANCISCO GUERRERO ${ }^{1}$ - FRANCISCO JiMÉNEZ-GÓMEZ ${ }^{1}$ - GEMA PARRA ${ }^{1}$ \\ ${ }^{1}$ Departamento de Biología Animal, Biología Vegetal y Ecología, Centro de Estudios \\ Avanzados de la Tierra (CEATierra), Universidad de Jaén, \\ Campus de las Lagunillas, s/n., 23071 Jaén, Spain \\ *Corresponding author \\ (phone: +34-953-213-054) \\ e-mail:aarco@ujaen.es \\ (Received $10^{\text {th }}$ Dec 2013; accepted 22 $2^{\text {nd }}$ July 2014)
}

\begin{abstract}
Intensive agricultural practices have been characterized by an overuse of agrochemicals. The inputs of chemicals in a watershed are likely to alter trophic interactions affecting its ecological integrity. This ecotoxicological study aims to identify warning signals of agrochemicals effects on a plankton community. Eighteen outdoor microcosms were used to establish an experiment with 2 concentrations of copper sulfate above and below the legal limit with six replicates lasting two weeks. Phytoplankton and Zooplankton structure changes were studied. Chlorophyll-a concentration was used as a functional indicator. A rapid change in phytoplankton structural features, abundance and size classes, was detected after both, below and above legal, concentrations. Similarly, Zooplankton structural changes showed an effect of copper exposures on abundance and composition. As Phytoplankton response was so rapid, it could be used as an early and direct warning signal, but also to warn of future indirect effects on zooplankton structural features owing to a change of food resources. In summary, the shifts across both trophic levels could be use as warning signals. Moreover, even legal limits do not protect the plankton community, which emphasises the need of a more ecotoxicological and realistic approach to achieve a balance between agriculture development and ecosystems conservation.
\end{abstract}

Key words: Copper; Microcosms; Plankton; Warning

\section{Introduction}

Current main environmental pressures are highly linked to human exponential population growth (United Nations Population Division 2000). One of the main consequences of population growth is an increase in food demand; therefore, agricultural practices intensify with the consequent increased use of agrochemicals (Valavanidis \& Vlachogianni 2010). The socio-economic value of agriculture cannot be denied, however, its integration with environmental criteria must be a priority. The improper use and / or application in excess of pesticides, herbicides and fertilizers generate an impact on the ecological integrity of ecosystems, affecting their structure and function (Troncoso et al. 2002, Parra et al. 2005). In this sense, there is enough bibliographic information which shows that intensive agriculture is causing the disappearance and / or pollution of wetlands (Casado \& Montes 1995, Troncoso et al. 2000, Parra et al, 2005, García-Muñoz et al. 2011). These impacts have consequences at different hierarchical levels: from the individual by morphological, physiological and biochemical alterations, to the community level through the loss of diversity, and impairing the value and services that healthy ecosystems provide (Montes \& Sala 2007).

Ecological indicators are tools which detect changes on ecosystems that are likely to impact ecological integrity and so then the community structure and function, and 
consequently ecosystem services. Structural features have been usually used alone to monitor and assess impacts on the ecosystem ecological integrity, focusing on communities assemblages and their resources. Structural attributes are easiest to visualize and they are, by far, the most commonly used. Examples are species numbers, dominant species, guild composition, taxonomic representation, abundances, size composition, and others (Bain et al. 2000). For instance, crustacean zooplankton size has been shown to be more strongly correlated with lake water quality than community taxonomic structure (Sprules 1984). In addition to structural levels, ecosystem functional aspects are gaining more importance in the evaluation of ecosystem integrity. Functional indicators assess rates and patterns of ecosystem processes and are considered to be an essential complementary aspect to assess ecological integrity due to their different sensibility from structural levels to environmental pressures (Gessner \& Chauvet 2002). However nowadays, society is demanding this to go further, with the intent of detecting, as soon as possible, the negative effects on ecosystems. In this sense, a warning signal is an important component of the integrated approaches that are needed to acquire a general knowledge of toxic impact, and which will allow predictions and early mitigation measures (Schmitt-Jansen et al. 2008).

The study is focused on the effects of a fungicide (copper sulfate) on trophic levels in wetlands which are surrounded by intensive olive tree agriculture. The aim was to evaluate how this toxic substance could alter structural and functional characteristics of the plankton components, and if these changes could be used as early warning signals. In the present study two different concentrations were used, the first above the legal limit, in order to find clear effects on the plankton community features. The second concentration, below the legal limit, in order to check if the changes could be detected even before the community was highly altered.

\section{Materials and methods}

\section{Microcosms}

Eighteen microcosms were set, based on, and adapted from, OECD (2006), and were placed outdoors in a specific installation at the University of Jaén (HUMEXPUJA, experimental wetland infrastructure in the University of Jaén, which were exposed to the same environmental conditions). Microcosms length, height and width were 0.34$0.28-0.24 \mathrm{~cm}$ respectively, 22.8 liters in volume and placed $15 \mathrm{~cm}$ apart from each other. Microcosms were filled with 18 liters of water and $5 \mathrm{~cm}$ of sediment. Water came from an artificial pond supply free of contamination and zooplankton (HUMEXPUJA). Sediment came from a natural wetland [Casillas wetland, UTM 30SVG1084 with a surface area of 2.2 ha. (Ortega et al. 2003)], it was homogenized and distributed among the microcosms. Microcosms were established in November 2011 and the experiment was finished in January 2012. There was a stabilization period of 7 weeks before adding copper to the microcosms in order to favour the development of the planktonic communities from the resistant structures present in the sediment. The experiment lasted 21 days, with a single pesticide spike on day 0 .

\section{Disturbance}

Control and two treatments of copper sulfate, with six replicates each one, were used in the experimental design. The first one, called high treatment $\left(\mathrm{H}: 0.2 \mathrm{mg} \mathrm{l}^{-1} \mathrm{Cu}\right)$, 
represents a concentration of copper sulphate over the limit established by both the Water Framework Directive (WFD 2000/60/CE) and its application into the Spanish National legislation (DMC 2000/60/CE) $\left(0.04 \mathrm{mg} \mathrm{l}^{-1}\right)$. The second one, low treatment (L: $0.02 \mathrm{mg} \mathrm{l}^{-1} \mathrm{Cu}$ ) shows a lower concentration than those legal limits previously mentioned. Therefore, our L treatment falls within legal limits, while the $\mathrm{H}$ treatment is one order of magnitude higher. Nominal dosages of copper sulfate were directly added and stirred over the water surface of the microcosms as an only pulse on day 0 for the whole experimental period. The criteria to establish the concentration of the treatments was not based on lethal concentration data of the species involved because the aim of the study focused on studying the effect over the entire plankton community. Water samples to control the fate of copper sulphate were taken every week and analyzed by ICP Mass Spectrometry.

\section{Physical-chemical variables}

Each microcosm was surveyed every seven days. Each time, physical-chemical measurements (temperature, $\mathrm{pH}, \%$ dissolved oxygen and conductivity) were taken using field probes. At the same time, water samples were taken, cold stored and transported to the laboratory to perform nitrogen dissolved nutrients (nitrate) and alkalinity analysis. Alkalinity was measured in the lab using a 848 Tritino Plus device. Nitrate was determined following the reduced column Cadmium method (Keeney \& Nelson 1982).

\section{Biological variables}

Abundance and changes in phytoplankton size distribution were evaluated with flow cytometry. Water samples were taken weekly, preserved in glutaraldehide (4\%), frozen in liquid nitrogen and stored at $80^{\circ} \mathrm{C}$ until running the analysis with BD- LSR Fortessa flow cytometer. Calibration spheres were used to obtain a cell size regression curve: $\mathrm{y}=$ $0.011 \mathrm{x}-14,388$, where " $\mathrm{x}$ " represents the mean of the Forward Scatter (FSC), and " $y$ " represents the cell size of the cells in $\mu \mathrm{m}^{3}$. Three cell size populations were determined characterized by a mean volume of $58 \mu \mathrm{m}^{3}$ (small), $304 \mu \mathrm{m}^{3}$ (medium) and $749 \mu \mathrm{m}^{3}$ (high). Population cells abundance were determined from an acquisition time of $180 \mathrm{~s}$ at a rate of $60 \mu \mathrm{L} \mathrm{min}^{-1}$. Data analysis was performed using the FACSDIVA software.

Chorophyll- $a$ concentration was measured weekly with a field fluorometer (Aquafluor deTurner Design). Chlorophyll-a (Chl-a) concentrations were later calculated using a previously obtained calibration curve determinate by fluorometry. Calibration samples were filtered through Whatman $\mathrm{GF} / \mathrm{C}$ glass microfibre filters $(1.2$ $\mu \mathrm{m}$ pore-size), and extracted in $90 \%$ acetone for $24 \mathrm{~h}$ at $4^{\circ} \mathrm{C}$ (Strickland \& Parsons 1968).

Zooplankton in microcosms was sampled weekly during the study through waterintegrated samples of $100 \mathrm{ml}$. Water integrated samples were collected, then filtered through a plankton net of $30 \mu \mathrm{m}$, and preserved in situ with formalin (4\%). The filtered water was returned to the microcosm. Zooplankton was identified to the lowest practical levels and abundance estimated.

Physical-chemical, plankton and Chl- $a$ variables were compared among microcosms using univariate and multivariate analyses with SPSS 19 software. Repeated measures of ANOVA were used to test for time and time $x$ treatment effects. An univariate ANOVA and a post hoc Tukey test at the sampling date were used to determine the 
significance of differences between treatments. Prior to analysis, data were tested for normality and homoscedasticity. Zooplankton data could not be treated with a parametric test due to its low abundance or even complete disappearance in some microcosms. Therefore, total zooplankton abundance, rotifera abundance, and copepod abundance were analyzed with the non-parametric test of Friedman to test for differences due to time and treatment. Wilcoxon post hoc test was also used to determinate which treatments were significantly different from one another. Ordination of treatment and control of physical-chemical parameters and biological variables, except phytoplankton cell size populations, were made considering a Principal Component Analysis (PCA) (CANOCO v4.5 software). PCA aimed understanding the main factors influencing microcosm's responses.

\section{Results}

Copper nominal concentrations were achieved with the spike on day 0 . The degradation was very low, therefore the average concentration exposure over the whole experiment matched the intended nominal concentrations (Table 1).

Table 1. Mean \pm standard deviation (S.D.) of copper sulfate after pulse, by the end of the experiment and the average concentration exposure.

\begin{tabular}{|l|l|l|l|}
\hline $\begin{array}{l}\text { Nominal concentration } \\
\left(\mathrm{mg} \mathrm{L}^{-1}\right)\end{array}$ & $\begin{array}{l}\text { Concentration }(\mathrm{mg} / \mathrm{l}) \\
\text { after pulse application } \\
(\text { day 0) } \pm \text { S.D. }\end{array}$ & $\begin{array}{l}\text { Concentration } \\
((\mathrm{mg} / \mathrm{l}) \text { after pulse } \\
(\text { day 14) } \pm \text { S.D. }\end{array}$ & $\begin{array}{l}\text { Average } \\
\text { concentration } \\
\text { exposure }(\mathrm{mg} / \mathrm{l}) \\
\pm \text { S.D. }\end{array}$ \\
\hline 0 & $0.01 \pm 0$ & $0.01 \pm 0$ & $0.01 \pm 0$ \\
\hline 0.02 & $0.03 \pm 0.01$ & $0.09 \pm 0.04$ & $0.06 \pm 0.05$ \\
\hline 0.20 & $0.11 \pm 0.09$ & $0.18 \pm 0.04$ & $0.14 \pm 0.07$ \\
\hline
\end{tabular}

Temperature ranged from $9^{\circ} \mathrm{C}$ to $13^{\circ} \mathrm{C}$ during the experimental period. Dissolved oxygen ( $\%$ DO), $\mathrm{pH}$, conductivity and alkalinity presented significant differences between treatments and controls, while nitrate concentration did not present significant differences among them, independently of the treatment (Table 2). At the same time, $\mathrm{pH}$ was higher in $\mathrm{H}$ treatments in day $0(\mathrm{~F}=396.820, \mathrm{P}=0.000)$ and in $\mathrm{L}$ and $\mathrm{H}$ treatments in day $7(\mathrm{~F}=236.197, \mathrm{P}=0.000)$. Dissolved Oxygen $(\%)$ was lower in $\mathrm{L}$ and $\mathrm{H}$ treatments from day $0(\mathrm{~F}=148.684, \mathrm{P}=0.000)$ till the end of the experiment in day7 $(\mathrm{F}=143.703, \mathrm{P}=0.000)$. Average oxygen content in controls, $\mathrm{L}$ and $\mathrm{H}$ treatments were 16,13 and $12 \mathrm{mg}^{-1}$ respectively. Conductivity was higher in $\mathrm{L}$ and $\mathrm{H}$ treatments from day $0(\mathrm{~F}=4.104, \mathrm{P}=0.038)$ till the end of the experiment in day7 $(\mathrm{F}=6.273, \mathrm{P}=$ 0.010). Alkalinity was higher in $\mathrm{L}$ and $\mathrm{H}$ treatments in day $7(\mathrm{~F}=43.707, \mathrm{P}=0.000)$. PCA shows that those differences were not relevant enough to discriminate among treatments. PCA of physical-chemical and biological variables discriminate the controls (to the left) from the treatments (to the right) (Fig. 1). The two main axes explain $89 \%$ of the variance, $\mathrm{x}$-axis explains $69 \%$ and $y$-axis explains $20 \%$ and they are correlated to conductivity and copper concentration and to rotifera and zooplankton abundance, respectively.

The results obtained show a negative effect of both copper sulphate concentrations tested on the plankton community under study. Even legal limits do not protect the 
plankton community. The plankton community was affected by a decrease in phytoplankton and zooplankton abundances under both copper concentrations.

Table 2. Physical-chemical and biological parameter measurements (mean $\pm S E$ ) in treatments and controls microcosms along the whole experiment period. ${ }^{*}$ Denotes statistical significant differences with the controls.

\begin{tabular}{|c|c|c|c|c|c|c|c|c|c|c|c|c|c|c|c|}
\hline 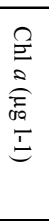 & 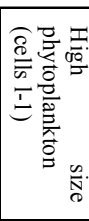 & 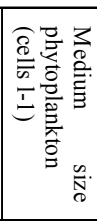 & 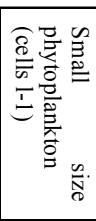 & 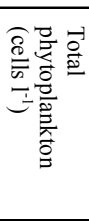 & 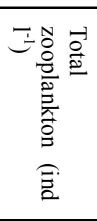 & 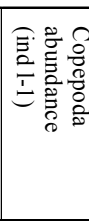 & 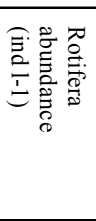 & 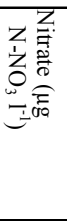 & $\begin{array}{l}\text { 窟 } \\
\text { 窟 }\end{array}$ & 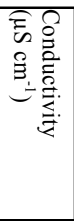 & $\dot{0}$ & $\bar{I}$ & 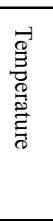 & 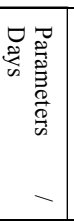 & 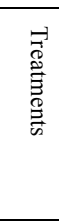 \\
\hline 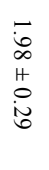 & $\begin{array}{l}\overline{\vec{\omega}} \\
\stackrel{*}{*}\end{array}$ & $\begin{array}{l}\bar{\nabla} \\
\stackrel{*}{*}\end{array}$ & $\begin{array}{l}\overrightarrow{\vec{u}} \\
\vec{*}\end{array}$ & $\begin{array}{l}\overrightarrow{\vec{u}} \\
\stackrel{*}{*}\end{array}$ & 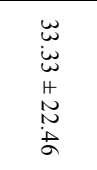 & 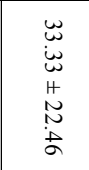 & 0 & $\begin{array}{l}0 \\
\dot{0} \\
\\
+ \\
\dot{8}\end{array}$ & 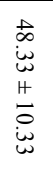 & $\begin{array}{l}\dot{0} \\
\dot{0} \\
0 \\
+ \\
\dot{0} \\
\dot{0}\end{array}$ & 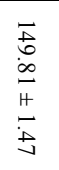 & $\begin{array}{l}0 \\
\dot{0} \\
\dot{H} \\
+ \\
\dot{\Delta}\end{array}$ & 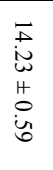 & 0 & $\begin{array}{l}\delta \\
\frac{\rho}{0} \\
\frac{\partial}{n}\end{array}$ \\
\hline $\begin{array}{l}\overrightarrow{0} \\
\overrightarrow{0} \\
0 \\
0 \\
0\end{array}$ & $\begin{array}{l}\overrightarrow{\vec{w}} \\
\stackrel{*}{*}\end{array}$ & $\begin{array}{l}\stackrel{0}{0} \\
\stackrel{*}{*}\end{array}$ & $\begin{array}{l}\widetilde{\sim} \\
\stackrel{*}{*} \\
\stackrel{\sim}{*}\end{array}$ & $\begin{array}{l}\infty \\
\infty \\
\stackrel{*}{*}\end{array}$ & $\begin{array}{l}\vec{a} \\
\dot{a} \\
H \\
\infty \\
\dot{u}\end{array}$ & 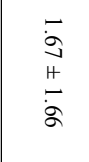 & $\begin{array}{l}\vec{u} \\
\ddot{8} \\
+ \\
\infty \\
\infty \\
\dot{a}\end{array}$ & $\begin{array}{l}\dot{0} \\
\dot{0} \\
0 \\
\dot{0}\end{array}$ & $\begin{array}{l}u \\
\infty \\
o \\
\dot{8} \\
+ \\
+ \\
\dot{4} \\
u\end{array}$ & $\begin{array}{l}\dot{0} \\
\dot{\infty} \\
\stackrel{+}{+} \\
\dot{0} \\
\dot{\omega}\end{array}$ & 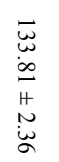 & $\begin{array}{l}\infty \\
\infty \\
\infty \\
\\
\dot{0} \\
0\end{array}$ & 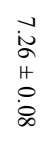 & $\checkmark$ & \\
\hline $\begin{array}{l}\omega \\
\dot{0} \\
+ \\
+ \\
\dot{0} \\
\text { aे }\end{array}$ & 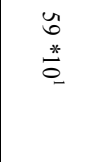 & $\begin{array}{l}\vec{J} \\
\stackrel{*}{0} \\
\text { w }\end{array}$ & $\begin{array}{l}\vec{Z} \\
\stackrel{*}{*}\end{array}$ & $\begin{array}{l}\text { U. } \\
\stackrel{*}{*}\end{array}$ & $\begin{array}{l}N \\
0 \\
\dot{O} \\
H \\
\stackrel{H}{H} \\
\dot{\omega}\end{array}$ & $\begin{array}{l}\vec{\omega} \\
\dot{\omega} \\
\dot{\omega} \\
+ \\
\infty \\
\infty \\
\infty\end{array}$ & $\begin{array}{l}\dot{\vec{g}} \\
\dot{\vec{u}} \\
\stackrel{\omega}{\omega} \\
\dot{\omega}\end{array}$ & $\begin{array}{l}\dot{0} \\
\dot{0} \\
+ \\
\dot{8}\end{array}$ & 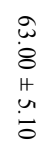 & $\begin{array}{l}\dot{0} \\
\dot{0} \\
4 \\
+ \\
\dot{0} \\
\dot{0}\end{array}$ & 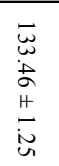 & $\begin{array}{l}\infty \\
\infty \\
\infty \\
1 \\
0 \\
\dot{\omega}\end{array}$ & $\begin{array}{l}\overrightarrow{0} \\
\dot{+} \\
+ \\
H \\
\dot{0} \\
\dot{\infty}\end{array}$ & F & \\
\hline 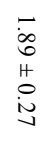 & $\begin{array}{l}\vec{\infty} \\
\stackrel{*}{*} \\
\stackrel{*}{i}\end{array}$ & $\begin{array}{l}\text { N } \\
\stackrel{n}{*} \\
\stackrel{*}{*}\end{array}$ & $\begin{array}{l}\vec{F} \\
\stackrel{\vec{*}}{\stackrel{*}{*}}\end{array}$ & $\begin{array}{l}\text { N } \\
\stackrel{*}{*} \\
\stackrel{*}{*}\end{array}$ & $\begin{array}{l}\tilde{N} \\
\dot{\sigma} \\
+ \\
\stackrel{\alpha}{\alpha}\end{array}$ & $\begin{array}{l}\vec{\infty} \\
\dot{\omega} \\
+ \\
+ \\
\infty \\
\vec{N}\end{array}$ & 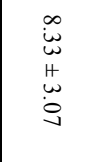 & $\begin{array}{l}\dot{8} \\
\dot{0} \\
\stackrel{+}{0} \\
\dot{8}\end{array}$ & 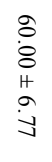 & $\begin{array}{l}0 \\
\infty \\
+ \\
+ \\
0 \\
\dot{0} \\
+\end{array}$ & 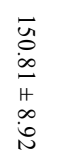 & $\begin{array}{l}\infty \\
\dot{0} \\
0 \\
ث \\
0 \\
\dot{0} \\
u\end{array}$ & $\begin{array}{l}\vec{\omega} \\
\dot{\omega} \\
H \\
0 \\
\dot{\omega} \\
\omega\end{array}$ & 0 & 宫 \\
\hline $\begin{array}{l}\stackrel{.}{0} \\
\infty \\
\stackrel{+}{\circ} \\
\stackrel{0}{0}\end{array}$ & $\begin{array}{l}\stackrel{\breve{*}}{*} \\
\stackrel{*}{\sigma}\end{array}$ & $\begin{array}{l}\text { N } \\
\text { * } \\
\text { 总 }\end{array}$ & 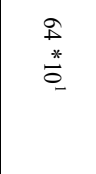 & 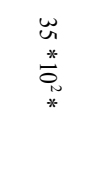 & 0 & 0 & 0 & $\begin{array}{l}8 \\
\dot{0} \\
+ \\
\dot{8} \\
8\end{array}$ & 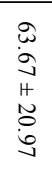 & $\begin{array}{l}0 \\
\dot{0} \\
\omega \\
+ \\
0 \\
1\end{array}$ & 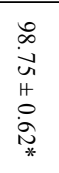 & 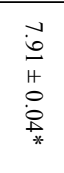 & $\begin{array}{l}\vec{D} \\
\dot{\infty} \\
\stackrel{+}{+} \\
\stackrel{+}{+}\end{array}$ & $\checkmark$ & \\
\hline $\begin{array}{l}\overrightarrow{\dot{y}} \\
\vec{H} \\
\stackrel{+}{+} \\
\dot{I}\end{array}$ & $\begin{array}{l} \pm \\
\text { ث } \\
\stackrel{*}{\sigma}\end{array}$ & 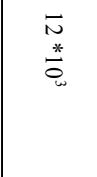 & 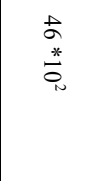 & $\begin{array}{l}\vec{\sigma} \\
\vec{*} \\
\vec{*} \\
*\end{array}$ & $\begin{array}{l}\omega \\
\dot{\omega} \\
\dot{\omega} \\
H \\
\omega \\
\dot{\omega} \\
\omega_{*} \\
*\end{array}$ & 0 & 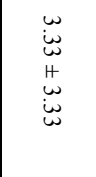 & $\begin{array}{l}\dot{0} \\
\dot{0} \\
+ \\
\dot{8}\end{array}$ & 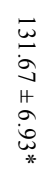 & 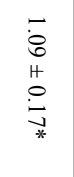 & 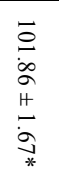 & 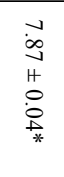 & 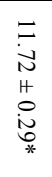 & $F$ & \\
\hline $\begin{array}{l}\vec{i} \\
\stackrel{N}{u} \\
\stackrel{+}{+} \\
\stackrel{\Delta}{N}\end{array}$ & $\begin{array}{l}\stackrel{8}{*} \\
\stackrel{*}{*}\end{array}$ & $\begin{array}{l}\stackrel{\leftrightarrows}{*} \\
\stackrel{*}{*}\end{array}$ & $\begin{array}{l}\stackrel{\varpi}{*} \\
\stackrel{*}{*}\end{array}$ & $\begin{array}{l}\underset{\infty}{u} \\
\stackrel{*}{*}\end{array}$ & 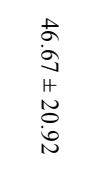 & $\begin{array}{l}\vec{a} \\
\dot{j} \\
+ \\
+\vec{u} \\
\dot{u}\end{array}$ & 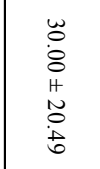 & $\begin{array}{l}0 \\
\dot{0} \\
+ \\
\\
0\end{array}$ & 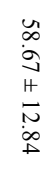 & 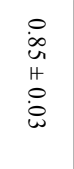 & 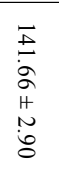 & $\begin{array}{l}\infty \\
\infty \\
0 \\
\ddot{1} \\
\stackrel{0}{0} \\
\dot{\omega}\end{array}$ & 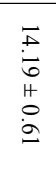 & 0 & 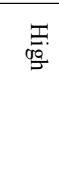 \\
\hline $\begin{array}{l}\dot{0} \\
\text { मे } \\
0 \\
\dot{0} \\
\dot{0}\end{array}$ & 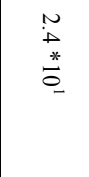 & $\begin{array}{l}\tilde{N} \\
\text { ** } \\
\text { O. }\end{array}$ & 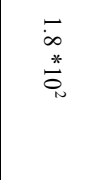 & 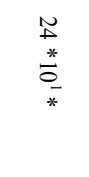 & $\begin{array}{l}\substack{\infty \\
\stackrel{\omega}{\omega} \\
+\infty \\
\infty \\
\omega \\
\omega} \\
\end{array}$ & 0 & 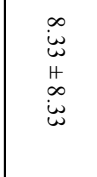 & $\begin{array}{l}0 \\
\dot{0} \\
+ \\
+ \\
\dot{8}\end{array}$ & $\begin{array}{l}\vec{b} \\
\dot{\vec{u}} \\
\dot{H} \\
\stackrel{\vec{\omega}}{\omega}\end{array}$ & $\begin{array}{l}0 \\
\dot{0} \\
0 \\
0 \\
\dot{0} \\
\dot{0}\end{array}$ & 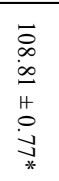 & 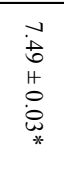 & 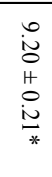 & $\checkmark$ & \\
\hline $\begin{array}{l}0 \\
\dot{8} \\
+ \\
0 \\
\dot{0}\end{array}$ & $\begin{array}{l}\tilde{N} \\
\stackrel{u}{*} \\
\stackrel{*}{0}\end{array}$ & $\begin{array}{l}\stackrel{t}{a} \\
\stackrel{*}{*} \\
\stackrel{i}{a}\end{array}$ & $\stackrel{\sim}{\stackrel{N}{*}}$ & $\begin{array}{l}\stackrel{9}{*} \\
\stackrel{*}{0} \\
\vec{*}\end{array}$ & $\stackrel{\circ}{*}$ & 0 & 0 & $\begin{array}{l}\dot{0} \\
\dot{0} \\
+ \\
\dot{0} \\
\dot{8}\end{array}$ & $\begin{array}{l}\stackrel{\vec{\sim}}{\tilde{\omega}} \\
\stackrel{\omega}{\omega} \\
+ \\
+ \\
+ \\
* \\
*\end{array}$ & $\begin{array}{l}\dot{0} \\
\dot{0} \\
H \\
0 \\
\dot{Q} \\
*\end{array}$ & $\begin{array}{l}\overrightarrow{5} \\
\dot{0} \\
\dot{8} \\
+ \\
\overrightarrow{9} \\
*\end{array}$ & $\begin{array}{l}\overrightarrow{0} \\
\dot{0} \\
4 \\
0 \\
\dot{8} \\
*\end{array}$ & $\begin{array}{l}\overrightarrow{\tilde{N}} \\
\dot{\omega} \\
H \\
\dot{0} \\
\dot{U} \\
*\end{array}$ & $\bar{F}$ & \\
\hline
\end{tabular}




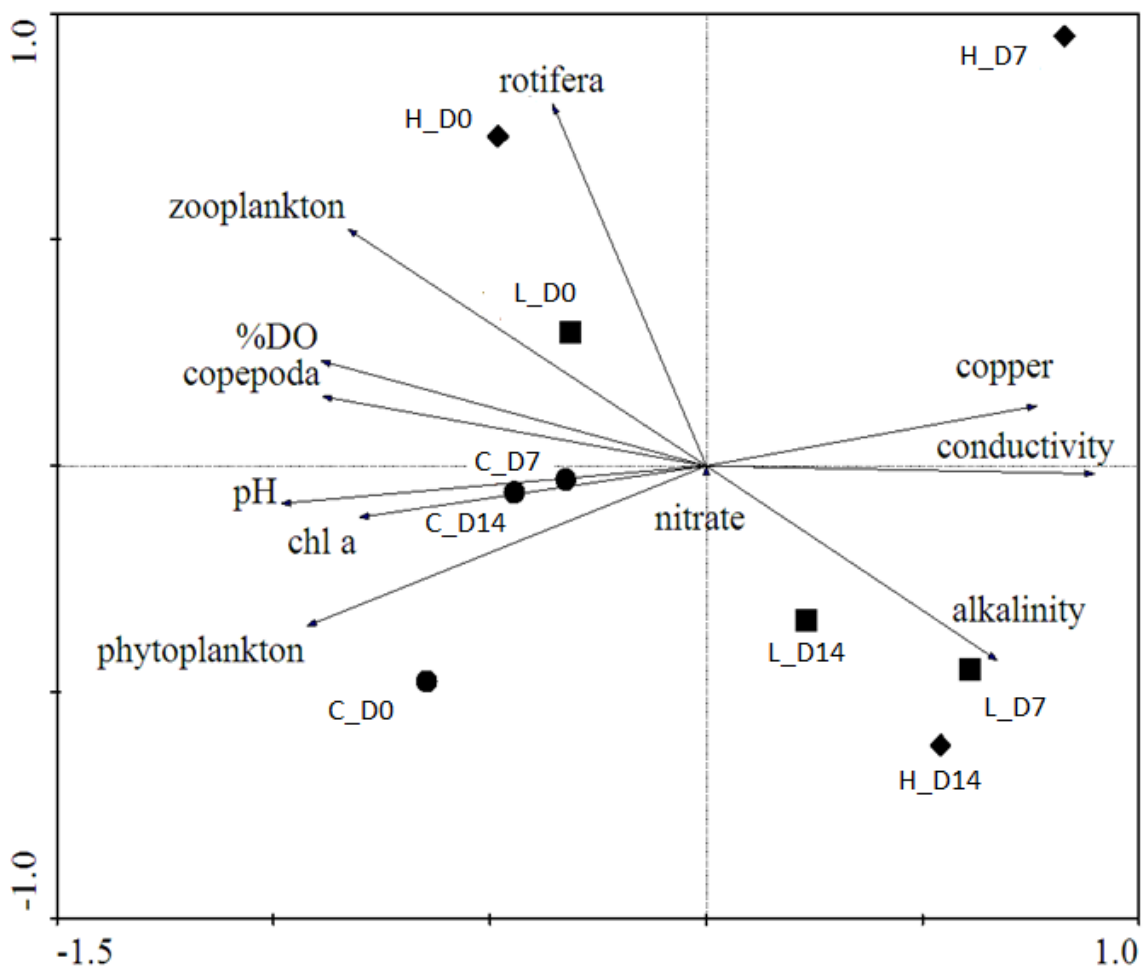

Figure 1. Physical-chemical PCA ordination graph. Arrows represent the lineal combination of zooplankton variables with the first and second axes. $C, L$ and $H$ stand for control, low and high copper treatments respectively. D indicates the sampling days.

Phytoplankton presented differences in population abundance among microcosms ( $\mathrm{F}$ $=5.447, \mathrm{P}=0.045$, Table 2). A drastic decrease of phytoplankton populations can be observed after the copper application in the treatments with respect to the control (Fig. 2 a). Chlorophyll $a$ did not show significant differences (day $0, \chi^{2}=2.648, \mathrm{P}=0.104$; day $7, \chi^{2}=2.406, \mathrm{P}=0.124$; day $14, \chi^{2}=2.351, \mathrm{P}=0.129 ;$ ) even though it was lower in microcosms treated with copper (Table 2). Our functional indicator, $\mathrm{Chl} a$, decreased but not significantly under both copper concentrations, in accordance with phytoplankton abundance decrease. In addition, variation of phytoplankton size classes of small, medium and high phytoplankton cells showed that copper treatment led to an increase in the small size group (Fig. 2).

Total zooplankton abundance was negatively affected mainly at the end of the experiment. The average abundance of total zooplankton during the study period was 23, 10 and 18 ind $1^{-1}$ in the control, $\mathrm{L}$ and $\mathrm{H}$ treatments, respectively. The zooplankton community was represented by the presence of rotifera (Euclanis sp., Brachionus sp. and Monostila sp.) and copedoda (Calanoida). Zooplankton abundance (Table 2) showed statistical differences among the controls and treatments at the end of the experiment $\left(\chi^{2}=9.500, P=0.009\right)$ and was lower in $\mathrm{L}$ treatment (Wilcoxon test $\mathrm{Z}=$ $2.060, P=0.039$,) and $\mathrm{H}$ treatment (Wilcoxon test, $\mathrm{Z}=-2.060, P=0.039$ ) than in controls. There were not statistically significant differences among zooplankton groups in control and treatments but they behaved in different ways. Copepods disappeared at the end of the experimental period, while the rotifers increased their abundance. 
a)

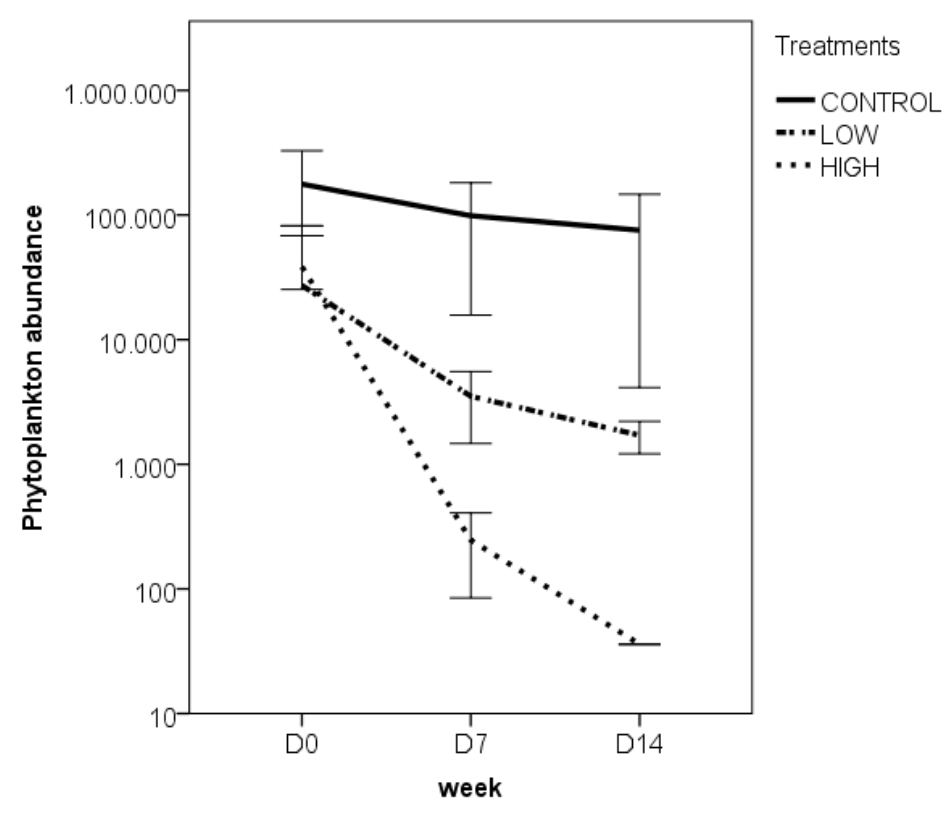

b)

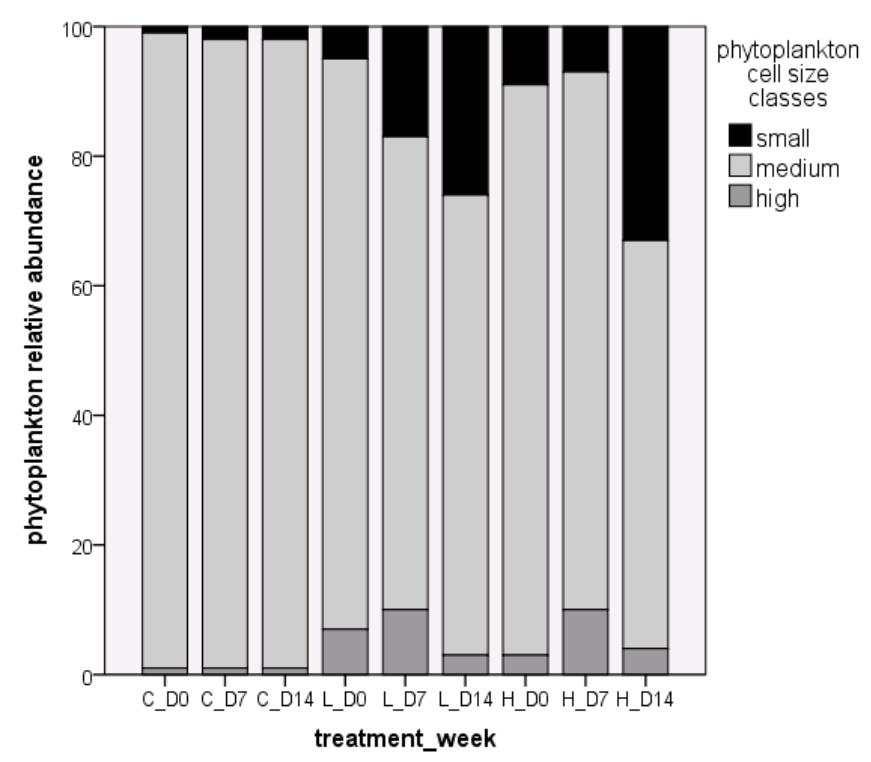

Figure 2. a) Phytoplankton abundance (cells L-1) and, b) cell class proportion (\%) along the experiment.

\section{Discussion}

Even though there are some specific differences in physical-chemical parameters during the experiment, PCA shows that those differences were not relevant enough to discriminate among treatments. Therefore, all microcosms were under the same water quality and an environmental condition which allows us to refute that community differences are neither related to water quality and environmental-induced differences among microcosms, but owing to treatment effects. 
Phytoplankton was highly affected during the whole experiment in both low and high treatments. Even working under legal copper concentrations, there are negative impacts on the aquatic community since phytoplankton abundance in treatments differed from the control abundance. Phytoplankton sensitivity to copper has been reported in other studies (Santos et al. 2002, Nayar et al. 2004). Moreover the changes in cell size group distribution showed by cytometry give information about the impairment in the plankton community and can be used as an early warning signal. Besides this fact, small size populations increased in microcosms treated with copper (Fig. 2 b). This implies a variation of predator-prey mass ratio. Body size relation is important in trophic interactions owing to its influence in growth efficiency. Growth efficiency depends on the relative body size of the prey, and on the prey density (Kerr \& Dickie 2001), and copper sulfate treatments have affected both aspects. Therefore, phytoplankton cell size changes towards smaller sizes may have indirect effects upon the zooplankton community through a reduction in its growth efficiency. Phytoplankton structural features showed the first warning signals: these were a drastic decrease of phytoplankton abundance and changes towards smallest cell sizes after copper application; hence, its measure could be used as a simple and efficient tool to identify an early impairment signal. In this sense, flow cytometry has been shown as a very rapid and useful technique. As has been mentioned before, a warning signal is an important component of the integrated approaches that are needed to acquire a general knowledge of toxic impact allowing predictions and early mitigation measures (Schmitt-Jansen et al. 2008) and flow cytometry could be easily incorporated in the assessment and biomonitoring programs.

The delay in zooplankton response could be related to an indirect effect of copper on trophic interactions. The direct effect of copper on phytoplankton affected zooplankton food availability. Therefore food resources decreased for zooplankton but the effect on zooplankton was not detected right away after copper application. Moreover, this different response timing is also related with the different life span of phytoplankton and zooplankton, being faster in phytoplankton. Even though there were no statistically significant differences among zooplankton groups in control and treatments, they behave in different way. For instance, the copepoda disappearance in some treatment microcosms suggest a specific impact in that group's ability to face the experimental conditions, and consequently in its potential recovery capacity. At the same time, rotifera responded differently to copper treatments, increasing its abundance in treatment microcosms, which implies that there had been a community shift both in $\mathrm{L}$ and $\mathrm{H}$ treatments that could not be observed at total zooplankton abundance level. In fact, the control microcosms had more than double that of the zooplankton in both $\mathrm{L}$ and $\mathrm{H}$ treatments, showing the importance of analyzing changes at a lower hierarchical level in order to better understand the changes at a higher hierarchical level. Going deeper into zooplankton shift, it has been shown in other studies that rotifera are more tolerant immediately after copper application than other organisms even up to $20 \mathrm{mg}^{-1}$ of copper, however after 8 days under copper exposure from $0.5 \mathrm{mg} \mathrm{l}^{-1}$ to $20 \mathrm{mg} \mathrm{l}^{-1}$ its population was dramatically affected (Källqvic \& Meadows 1978). Large-bodied zooplankton also is more sensitive to environmental stressors including pesticides than their smaller congeners (Havens \& Hanazato 1993). Further, copedoda and rotifera play a different role in the ecosystems and in the food web structure. For instance, macrozooplankton, as copedoda, grazing pressure has a stronger role than rotifera in regulating phytoplankton which is an important function to control eutrophication 
(Miracle et al. 2007). Kasai \& Hanazato (1995), using experimental ponds, observed that the herbicide simetryn caused a decrease in zooplankton density due to indirect effects related to a decrease of algae. But picking up the changes in phytoplankton size, it is interesting to note that at the end of the experiments, both $\mathrm{L}$ and $\mathrm{H}$ treatments, showed higher proportion of small phytoplankton cells than control. The smallest filterfeeders could take advantage exploiting the mentioned small food resources, increasing their abundances. In addition, the community shift could be a response caused not only by the apparent higher rotifera tolerance to copper but also by other indirect situations. The main indirect effect is the decrease of competence for food resources due to copedoda reduction that allows the increase of rotifera population. Miracle et al. (2007) found an inverse relationship among rotifera and cyclopoida copepod abundance under perturbation. This inverse relationship of rotifera and copedoda under perturbation has been found in other studies. For instance Richard et al. (1985) observed how under herbicide treatment there were shifts from copedoda and copedoda-cladoceran dominated communities to rotifera and small cladoceran dominated communities. A similar relationship was found by Gagneten \& Paggi (2009), under heavy metals treatments $(\mathrm{Pb}$ and $\mathrm{Cu})$ rotifera increased while copedoda and cladocera decreased. Both studies used such relationship as a tool to characterize the water bodies under study: in the first case the trend towards rotifera was identified as an indicator of eutrophy impairment and in the second case as a tool to determinate heavy metals impairment. In this study, an inverse relationship between rotifera and copedoda matches with other observations that indicate copper impairment as the decrease of total zooplankton in the treatments. Therefore, it supports its use as an easy and cost-efficient indicator and warning signal of contamination in aquatic systems as Gagneten \& Paggi (2009) also suggested. The negative impact that has been showed during this short term experiment on the poorest food resources could be intensified, in the long term, to the zooplankton community. The impairment in trophic relationships observed due to copper exposition, allows considering both, phytoplankton and zooplankton changes, as early warning signals. In nature, loss of species at basal trophic levels can affect production at higher levels and thus can also lead to decreased energy transfer efficiency (Gamfeldt et al. 2005). Undoubtedly, further studies must be developed to confirm these results in long term exposition, with different toxic substances, and this with holistic approaches that can detect indirect effect and alarm signals as the phytoplankton did.

Chlorophyll content is used to highlight stress due to a single environmental factor or to a combination of different environmental factors, but it also constitutes potential biomarkers of anthropogenic stress (Ferrat et al. 2003). However, in the present study Chlorophyll- $a$, as a functional indicator, has not been shown as effective as structural changes indicating alterations in plankton community.

This study works towards a deeper understanding of the agrochemicals negative effects on plankton communities at concentrations above but also below their legal limits. Surprisingly, even legal limits do not protect the plankton community. This result emphasizes the need of more ecological and realistic approaches to ensure adequate regulation limits in order to achieve a balance between development and conservation.

Acknowledgements. We would like to thank Francisco Márquez for providing fieldwork devices, José Robles for his valuable technical advices for copper analysis and Adrian Green for the English Grammar revision. This research has been partly supported by a grant from the University of Jaén (Spain) to Ana 
Isabel Del Arco Ochoa and the research group of Ecología y Biodiversidad de Sistemas Acuáticos (RNM-300, Spain).

\section{REFERENCES}

[1] Bain, M. B., Harig, A. L., Loucks, D. P., Goforth, R. R.,. Millsa, K.E. (2000): Aquatic ecosystem protection and restoration: advances in methods for assessment and evaluation. - Environ. Sci. Policy 3: S89-S98.

[2] Casado, S., Montes, C. (1995): Guide of Spanish lakes and wetlands, First ed. J.M. Reyero, Madrid.

[3] Ferrata, L., Pergent-Martinia, C. \& Roméo, M. (2003): Assessment of the use of biomarkers in aquatic plants for the evaluation of environmental quality: application to seagrasses. - Aquat. Toxicol. 65:187-204.

[4] Gessner, M.O., Chauvet, E. (2002): A case for using litter breakdown to assess functional stream integrity. - Ecol. Appl. 12: 498-510.

[5] Gagneten, A. M. \& Paggi, J. C. (2009): Caracterización fisicoquímica y ecotoxicológica de un ambiente fluvial contaminado por metales pesados en el centro-este de Argentina. IX Congreso de la Sociedad de Química y Toxicología Ambiental de Latinoamérica (SETAC LA) -II Congreso SETAC PERU.

[6] Gamfeldt, L., Hillebrand, H. \& Jonsson, P. R., 2005: Species richness changes across two trophic levels simultaneously affect prey and consumer biomass. - Ecol. Let., 8: 696-703.

[7] García-Muñoz, E., Guerrero, F., Parra, G. (2011): Effects of previous sublethal pulse to ammonium nitrate on mortality and total length on Epidalea calamita larvae.- Chem. Ecol. 84: 671-675

[8] Havens, K. E. \& Hanazato, T. (1993): Zooplankton responses to chemical stressors: a comparison of results from acidification and pesticide contamination research. - Environ Pollut. 82:277-288.

[9] Kallqvist, T., Meadows, B.S. (1978): The toxic effect of copper on algae and rotifers from soda lake (Lake Nakuru, East Africa).- Water Res. 12: 771-775

[10] Kasai, F. \& Hanazato, T. (1995): Effects of the triazine herbicide, simetryn, on freshwater plankton communities in experimental ponds. - Environ. Poll. 89:197-202.

[11] Keeney, D. R. \& Nelson D. W., 1982: Nitrogen-inorganic forms. Methods of soil analysis. Part 2 -Chemical and microbiological properties. - Am. Soc. Agron. 9:643-698.

[12] Kerr, S. R \& Dickie, L. M. (2001): The biomass spectrum. A predator-prey theory of aquatic production. In Columbia University Press (ed.) - New York, pp. $1-352$.

[13] Miracle, M. R., Alfonso, M. T. \& Vicente, E. (2007): Fish and nutrient enrichment effects on rotifera in a Mediterranean shallow lake: a mesocosm experiment. - Hydrobiologia 593:77-94.

[14] Montes, C., Sala, O. (2007): La evaluación de los ecosistemas del milenio. Las relaciones entre el funcionamiento de los ecosistemas y el bienestar humano. - Ecosistemas 16: 137147.

[15] Nayar, S., Goh, B. P. L. \& Chou, L. M. (2004): Environmental impact of heavy metals from dredged and resuspended sediments on phytoplankton and bacteria assessed in in situ mesocosms. - Ecotoxicol. Environ. Saf. 59:349-369.

[16] OECD (2006): Guidance document on simulated freshwater lentic field tests (outdoor microcosms and mesocosms). - OECD series on testing and assessment, Guideline number 53.

[17] Ortega, F., Parra, G. \& Guerrero, F. (2003): Los humedales del alto Guadalquivir: inventario, tipologías y estado de conservación, First ed. Ecología, manejo y conservación de los humedales. - Colección Actas, 49. Instituto de Estudios Almerienses (Diputación de Almería). Almería 49:113-123. 
[18] Parra, G., Jiménez-Melero, R., Guerrero, F. (2005): Agricultural impacts on Mediterranean wetlands: the effect of pesticides on survival and hatching rates in copepods.- Int. J. Limnol. 41: 161-167.

[19] Richard, D. I., Small, J. W. \& Osborne J. A. (1985): Response of zooplankton to the reduction and elimination of submerged vegetation by grass carp and herbicide in four Florida lakes. -Hydrobiologia 123:97-108.

[20] Santos, M. M., Garrido, I. M., Goncalves, F., Soares A. M. V. M. \& Ribeiro, R. (2002): An in situ bioassay for estuarine environments using the microalga Phaeodactylum tricornutum. -Environ. Toxicol. Chem. 21:567-574.

[21] Schmitt-Jansen, M., Veit, U., Dudel G. \& Altenburger, R. (2008): An ecological perspective in aquatic ecotoxicology: approaches and challenges. - Basic Appl. Ecol. 9:337-345.

[22] Sprules, W. G. (1984): Towards an optimal classification of zooplankton for lake ecosystem studies. - Verh. Internat. Verein. Limnol. 22:320-325.

[23] Strickland, J.D.H. \& Parsons, T.R., 1968: A practical handbook of seawater analysis. Ottawa: fisheries research board of Canada. - Bulletin 167, 293 pp.

[24] Troncoso, L., Galleguillos, R., Larrain, A. (2000): Effects of copper on the fitness of the Chilean scallop Argopecten purpuratus (Mollusca: Bivalvia). - Hydrobiologia 420, 185 189.

[25] United Nations Population Division (2005): Urban and rural areas 2005. - United Nations. New York, USA.

[26] Valavanidis, A., Vlachogianni, TH. (2010): Agricultural Pesticides: Ecotoxicologial studies and environmental risk assessment. - Sci. Advances Environ. Toxicol. Ecotoxicol. Issues 1-15.

[27] Water Framework Directive, (2000): Water Framework Directive of the European Parliament and the Council, of 23 October 2000, establishing a framework for Community action in the field of water policy. - Off. J. Eur. Communities 327:1-72. 